\title{
STABILITY INEQUALITIES FOR PROJECTIONS OF CONVEX BODIES
}

\author{
ALEXANDER KOLDOBSKY
}

\begin{abstract}
The projection function $P_{K}$ of an origin-symmetric convex body $K$ in $\mathbb{R}^{n}$ is defined by $P_{K}(\xi)=|K| \xi^{\perp} \mid, \quad \xi \in S^{n-1}$, where $K \mid \xi^{\perp}$ is the projection of $K$ to the central hyperplaner $\xi^{\perp}$ perpendicular to $\xi$, and $|K|$ stands for volume of proper dimension.

We prove several stability and separation results for the projection function. For example, if $D$ is a projection body in $\mathbb{R}^{n}$ which is in isotropic position up to a dilation, and $K$ is any originsymmetric convex body in $\mathbb{R}^{n}$ such that that there exists $\xi \in S^{n-1}$ with $P_{K}(\xi)>P_{D}(\xi)$, then

$$
\max _{\xi \in S^{n-1}}\left(P_{K}(\xi)-P_{D}(\xi)\right) \geq \frac{c}{\log ^{2} n}\left(|K|^{\frac{n-1}{n}}-|D|^{\frac{n-1}{n}}\right),
$$
\end{abstract}

where $c$ is an absolute constant.

As a consequence, we prove a hyperplane inequality

$$
S(D) \leq C \log ^{2} n \max _{\xi \in S^{n-1}} S\left(D \mid \xi^{\perp}\right)|D|^{\frac{1}{n}},
$$

where $D$ is a projection body in isotropic position, up to a dilation, $S(D)$ is the surface area of $D$, and $C$ is an absolute constant. The proofs are based on the Fourier analytic approach to projections developed in [KRZ].

\section{INTRODUCTION}

Let $K$ be an origin-symmetric convex body in $\mathbb{R}^{n}$. The projection function $P_{K}: S^{n-1} \rightarrow(0, \infty)$ of $K$ is defined for every $\xi \in S^{n-1}$ as the $(n-1)$-dimensional volume of the orthogonal projection of $K$ to the central hyperplane $\xi^{\perp}$ perpendicular to $\xi$. We write

$$
P_{K}(\xi)=|K| \xi^{\perp} \mid, \quad \forall \xi \in S^{n-1},
$$

where $K \mid \xi^{\perp}$ is the projection of $K$ to $\xi^{\perp}$, and $|K|$ stands for volume of proper dimension.

The classical uniqueness theorem of Aleksandrov [A] states that every origin-symmetric convex body is uniquely determined by the function $P_{K}$; see also [G, Theorem 3.3.6]. The corresponding volume comparison question was posed by Shephard [Sh] in 1964. Suppose that $K, D$ 
are origin-symmetric convex bodies in $\mathbb{R}^{n}$, and $P_{K}(\xi) \leq P_{D}(\xi)$ for every $\xi \in S^{n-1}$. Does it necessarily follow that $|K| \leq|D|$ ? Shephard's problem was solved by Petty [Pe] and Schneider [S1], independently, and the answer is affirmative only in dimension $n=2$. Both solutions were based on a connection with projection bodies (see definition in Section 2), as follows. If $D$ is a projection body, the answer to Shephard's question is affirmative for every $K$. On the other hand, if $K$ is not a projection body, one can construct $D$ giving together with $K$ a counterexample. The final answer to the problem follows from the fact that only in dimension $n=2$ all origin-symmetric convex bodies are projection bodies.

For the affirmative cases in volume comparison problems, stability and separation problems were proposed in [K2]. Suppose that $\varepsilon>0$, $K, L$ are origin-symmetric convex bodies in $\mathbb{R}^{n}$, and $L$ is a projection body. The stability problem asks whether there exists a constant $c>0$ not dependent on $K, L$ or $\varepsilon$ and such that the inequalities $P_{K}(\xi) \leq$ $P_{L}(\xi)+\varepsilon$, for all $\xi \in S^{n-1}$, imply $|K|^{\frac{n-1}{n}} \leq|L|^{\frac{n-1}{n}}+c \varepsilon$. The separation problem asks whether inequalities $P_{K}(\xi) \leq P_{L}(\xi)-\varepsilon$, for all $\xi \in S^{n-1}$, imply $|K|^{\frac{n-1}{n}} \leq|L|^{\frac{n-1}{n}}-c \varepsilon$, again with a constant $c>0$ not dependent on $K, L, \varepsilon$.

Separation was proved in [K2] (see also [K3], where the constant is written precisely) with the best possible constant

$$
c=c_{n}=\frac{\left|B_{2}^{n}\right|^{\frac{n-1}{n}}}{\left|B_{2}^{n-1}\right|}, c_{n} \in\left(\frac{1}{\sqrt{e}}, 1\right),
$$

where $B_{2}^{n}$ is the unit Euclidean ball in $\mathbb{R}^{n}$. Stability was proved in [K2] with constants dependent on the bodies, using rough estimates for $M$ and $M^{*}$ parameters of convex bodies. In this article we prove stability with constants not dependent on the bodies, but under an additional assumption that $L$ is in isotropic position, up to a dilation; see Proposition 1 . To do this, we use a recent $M^{*}$-estimate of Milman [M]. We also prove that, if the projection body condition is dropped, one can get results going in the direction opposite to stability and separation. Namely, we give examples of origin-symmetric convex bodies $K, L$ such that $P_{K} \leq P_{L}+\varepsilon$, but $|K|^{\frac{n-1}{n}} \geq|L|^{\frac{n-1}{n}}+c \varepsilon$, and also examples of $K, L$ where $P_{K} \leq P_{L}-\varepsilon$, but $|K|^{\frac{n-1}{n}} \geq|L|^{\frac{n-1}{n}}-c \varepsilon$, with $c$ not dependent on the bodies or small enough $\varepsilon$. In some sense, these results provide a quantitative version of the solution to Shephard's problem.

Stability and separation immediately imply what we call volume difference inequalities. In fact, if stability holds and there exists $\xi \in S^{n-1}$ such that $P_{K}(\xi)>P_{L}(\xi)$, then we put $\varepsilon=\max _{\xi \in S^{n-1}}\left(P_{K}(\xi)-P_{L}(\xi)\right)$, 
and get

$$
|K|^{\frac{n-1}{n}}-|L|^{\frac{n-1}{n}} \leq c \max _{\xi \in S^{n-1}}\left(P_{K}(\xi)-P_{L}(\xi)\right) .
$$

Similarly, if separation holds, we get

$$
|L|^{\frac{n-1}{n}}-|K|^{\frac{n-1}{n}} \geq c \min _{\xi \in S^{n-1}}\left(P_{L}(\xi)-P_{K}(\xi)\right) .
$$

We provide such an inequality for the stability result mentioned above; see Theorem 1.

Volume difference inequalities lead to hyperplane inequalities for surface area of projection bodies. It was proved in [K3] that if $D$ is a projection body in $\mathbb{R}^{n}$, then

$$
S(D) \geq c \min _{\xi \in S^{n-1}} S\left(D \mid \xi^{\perp}\right)|D|^{\frac{1}{n}},
$$

where $S(D)$ is the surface area of $D$, and $c$ is an absolute constant. In this paper we prove an inequality that complements (1). If $D$ is a projection body in isotropic position, up to a dilation, then

$$
S(D) \leq C \log ^{2} n \max _{\xi \in S^{n-1}} S\left(D \mid \xi^{\perp}\right)|D|^{\frac{1}{n}}
$$

where $C$ is an absolute constant; see Theorem 2 below.

Finally, we show that if the condition that $D$ is a projection body is removed, volume difference inequalities can go in the opposite direction; see Theorems 3 and 4 .

We extensively use the Fourier analytic approach to projections of convex bodies developed in [KRZ]; see also [K1, Chapter 8].

\section{STABILITY THEOREMS}

We need several definitions from convex geometry. We refer the reader to [S2] for details.

The support function of a convex body $K$ in $\mathbb{R}^{n}$ is defined by

$$
h_{K}(x)=\max _{\left\{\xi \in \mathbb{R}^{n}:\|\xi\|_{K}=1\right\}}(x, \xi), \quad x \in \mathbb{R}^{n} .
$$

If $K$ is origin-symmetric, then $h_{K}$ is a norm on $\mathbb{R}^{n}$.

The surface area measure $S(K, \cdot)$ of a convex body $K$ in $\mathbb{R}^{n}$ is a measure on $S^{n-1}$ defined as follows. For every Borel set $E \subset S^{n-1}$, $S(K, E)$ is equal to Lebesgue measure of the part of the boundary of $K$ where normal vectors belong to $E$. We usually consider bodies with absolutely continuous surface area measures. A convex body $K$ is said to have the curvature function $f_{K}: S^{n-1} \rightarrow \mathbb{R}$ if its surface 
area measure $S(K, \cdot)$ is absolutely continuous with respect to Lebesgue measure $\sigma_{n-1}$ on $S^{n-1}$, and

$$
\frac{d S(K, \cdot)}{d \sigma_{n-1}}=f_{K} \in L_{1}\left(S^{n-1}\right),
$$

so $f_{K}$ is the density of $S(K, \cdot)$.

The volume of a body can be expressed in terms of its support function and curvature function:

$$
|K|=\frac{1}{n} \int_{S^{n-1}} h_{K}(x) d S(K, x)=\frac{1}{n} \int_{S^{n-1}} h_{K}(x) f_{K}(x) d x,
$$

with the latter equality if $f_{K}$ exists.

If $K$ and $L$ are two convex bodies in $\mathbb{R}^{n}$, the mixed volume $V_{1}(K, L)$ is equal to

$$
V_{1}(K, L)=\frac{1}{n} \lim _{\varepsilon \rightarrow+0} \frac{|K+\epsilon L|-|K|}{\varepsilon} .
$$

The first Minkowski inequality (see for example [K1, p.23]) asserts that for any convex bodies $K, L$ in $\mathbb{R}^{n}$,

$$
V_{1}(K, L) \geq|K|^{\frac{n-1}{n}}|L|^{\frac{1}{n}}
$$

Mixed volume can also be expressed in terms of the support and curvature functions:

$$
V_{1}(K, L)=\frac{1}{n} \int_{S^{n-1}} h_{L}(x) d S(K, x)=\frac{1}{n} \int_{S^{n-1}} h_{L}(x) f_{K}(x) d x .
$$

Let $K$ be an origin-symmetric convex body in $\mathbb{R}^{n}$. The projection body $\Pi K$ of $K$ is defined by

$$
h_{\Pi K}(\theta)=|K| \theta^{\perp} \mid, \quad \forall \theta \in S^{n-1} .
$$

If $L$ is the projection body of some convex body, we simply say that $L$ is a projection body. We refer the reader to [K1, Chapter 8] for necessary information about projection bodies. We just mention here that, by a result of Bolker [Bl], an origin-symmetric convex body is a projection body if and only if its polar body is the unit ball of a subspace of $L_{1}$. The unit balls of the spaces $\ell_{p}^{n}, p \geq 2$ are projection bodies, while the unit balls of $\ell_{p}^{n}, p<2, n \geq 3$ are not. There are other examples of bodies in $\mathbb{R}^{n}, n \geq 3$ that are not projection bodies; see $[\mathrm{S} 2]$ or $[\mathrm{K} 1]$.

The classes of projection bodies $K$ for which the functions $h_{K}$ and $f_{K}$ are infinitely smooth are dense in the class of all projection bodies in the Hausdorff metric; see [S2, p.151] and [GZ]. The class of projection 
bodies for which the curvature function is strictly positive is also dense in the class of all projection bodies; see [GZ] or [K1, p.158, p. 161].

We say that a body $K$ is in isotropic position if $|K|=1$ and there exists a constant $L_{K}>0$ such that

$$
\int_{K}(x, \xi)^{2} d x=L_{K}^{2}, \quad \forall \xi \in S^{n-1} .
$$

For every origin-symmetric convex body $K$ in $\mathbb{R}^{n}$ there exists $T \in G L_{n}$ such that $T K$ is in isotropic position. The constant $L_{K}$ is called the isotropic constant of $K$. It is known that $L_{K} \geq L_{B_{2}^{n}}$ for every symmetric convex body $K$ in $\mathbb{R}^{n}$; see [MP, p.93]. The question of whether $L_{K}$ is bounded from above by an absolute constant is the matter of the well-known and still open slicing problem. The best known estimate $L_{K} \leq O\left(n^{1 / 4}\right)$ is due to Klartag [Kl], who improved an earlier estimate of Bourgain [Bo]. We refer the reader to [BGVV] for these results and more about the isotropic position and the slicing problem.

We use the Fourier approach to projections of convex bodies developed in [KRZ]; see also [K1, Chapter 8]. We consider Schwartz distributions, i.e. continuous functionals on the space $\mathcal{S}\left(\mathbb{R}^{n}\right)$ of rapidly decreasing infinitely differentiable functions on $\mathbb{R}^{n}$. The Fourier transform of a distribution $f$ is defined by $\langle\hat{f}, \phi\rangle=\langle f, \hat{\phi}\rangle$ for every test function $\phi \in \mathcal{S}\left(\mathbb{R}^{n}\right)$. For any even distribution $f$, we have $(\hat{f})^{\wedge}=(2 \pi)^{n} f$.

For $f \in C^{\infty}\left(S^{n-1}\right)$ and $p=1$ or $p=-n-1$, we denote by

$$
\left(f \cdot r^{p}\right)(x)=f\left(x /|x|_{2}\right)|x|_{2}^{p}
$$

the extension of $f$ to a homogeneous function of degree $p$ on $\mathbb{R}^{n}$. By [K1, Lemma 3.16], there exists $g \in C^{\infty}\left(S^{n-1}\right)$ such that

$$
\left(f \cdot r^{p}\right)^{\wedge}=g \cdot r^{-n-p} .
$$

In particular, if the support function $h_{K}$ is infinitely smooth on $S^{n-1}$, then the Fourier transform of $h_{K} \cdot r$ is an infinitely smooth function $g$ on the sphere extended to a homogeneous function of degree $-n-1$ on $\mathbb{R}^{n} \backslash\{0\}$. In this case we simply write $\left(h_{K} \cdot r\right)^{\wedge}(\theta)$ for $\theta \in S^{n-1}$, meaning the function $g$. It was proved in [KRZ] (see also [K1, Theorem 8.6]) that an origin-symmetric convex body $K$, for which $h_{K}$ is infinitely smooth, is a projection body if and only if

$$
\left(h_{K} \cdot r\right)^{\wedge}(\theta) \leq 0, \quad \forall \theta \in S^{n-1} .
$$

It was also proved in [KRZ] (see also [K1, Theorem 8.2]) that if an origin-symmetric body $K$ has curvature function $f_{K}$, then

$$
\left(f_{K} \cdot r^{-n-1}\right)^{\wedge}(\theta)=-\pi|K|_{\theta^{\perp}} \mid=-\pi P_{K}(\theta), \quad \forall \theta \in S^{n-1} .
$$


The following version of Parseval's formula was proved in [KRZ] (see also [K1, Lemma 8.8]). If $K, L$ are origin-symmetric convex bodies, $K$ has infinitely smooth support function and $L$ has infinitely smooth curvature function, then

$$
\int_{S^{n-1}}\left(h_{K} \cdot r\right)^{\wedge}(\xi)\left(f_{L} \cdot r^{-n-1}\right)^{\wedge}(\xi) d \xi=(2 \pi)^{n} \int_{S^{n-1}} h_{K}(x) f_{L}(x) d x
$$

Lemma 1. Let $K$ be an origin-symmetric convex body in $\mathbb{R}^{n}$ such that the support function $h_{K}$ is infinitely smooth. Then

$$
\int_{S^{n-1}}\left(h_{K} \cdot r\right)^{\wedge}(\xi) d \xi \leq-\frac{(2 \pi)^{n} n}{\pi} c_{n}|K|^{1 / n}
$$

Recall that $c_{n}=\left|B_{2}^{n}\right|^{\frac{n-1}{n}} /\left|B_{2}^{n-1}\right| \in\left(\sqrt{\frac{1}{e}}, 1\right)$.

Proof : The curvature function of the unit Euclidean ball $B_{2}^{n}$ is constant, $f_{2} \equiv 1$. By $(8)$,

$$
\left(f_{2} \cdot r^{-n-1}\right)^{\wedge}(\xi)=-\pi\left|B_{2}^{n-1}\right|, \quad \forall \xi \in S^{n-1} .
$$

By (4), (3) and (9),

$$
\begin{aligned}
& \int_{S^{n-1}}\left(h_{K} \cdot r\right)^{\wedge}(\xi) d \xi=-\frac{1}{\pi\left|B_{2}^{n-1}\right|} \int_{S^{n-1}}\left(h_{K} \cdot r\right)^{\wedge}(\xi)\left(f_{2} \cdot r^{-n-1}\right)^{\wedge}(\xi) d \xi \\
& =-\frac{(2 \pi)^{n}}{\pi\left|B_{2}^{n-1}\right|} \int_{S^{n-1}} h_{K}(x) f_{2}(x) d x \leq-\frac{(2 \pi)^{n} n|K|^{1 / n}\left|B_{2}^{n}\right|^{(n-1) / n}}{\pi\left|B_{2}^{n-1}\right|} .
\end{aligned}
$$

Lemma 2. Let $K$ be an origin-symmetric convex body in $\mathbb{R}^{n}$ such that the support function $h_{K}$ is infinitely smooth and $K$ is a dilate of an isotropic body. Then

$$
\int_{S^{n-1}}\left(h_{K} \cdot r\right)^{\wedge}(\xi) d \xi \geq-C(2 \pi)^{n} n \log ^{2}(1+n) L_{K}|K|^{1 / n},
$$

where $C$ is an absolute constant.

Proof : By the same argument as in Lemma 1 (recall that $f_{2} \equiv 1$ ) we have

$$
\int_{S^{n-1}}\left(h_{K} \cdot r\right)^{\wedge}(\xi) d \xi=-\frac{(2 \pi)^{n}}{\pi\left|B_{2}^{n-1}\right|} \int_{S^{n-1}} h_{K}(x) d x
$$

Now use the following estimate of E.Milman [M, Theorem 1.1]

$$
\frac{1}{\left|S^{n-1}\right|} \int_{S^{n-1}} h_{K}(x) d x \leq C_{1} \sqrt{n} \log ^{2}(1+n) L_{K}|K|^{1 / n},
$$

where $C_{1}$ is an absolute constant, and note that $\left|S^{n-1}\right| /\left|B_{2}^{n-1}\right| \sim \sqrt{n}$ to get the result. 
We now prove stability in the affirmative direction of Shephard's problem under the additional condition that the body $D$ is a dilate of an isotropic body.

Proposition 1. Suppose that $\varepsilon>0, K$ and $D$ are origin-symmetric convex bodies in $\mathbb{R}^{n}$, and $D$ is a projection body which is a dilate of an isotropic body. If for every $\xi \in S^{n-1}$

$$
P_{K}(\xi) \leq P_{D}(\xi)+\varepsilon
$$

then

$$
|K|^{\frac{n-1}{n}} \leq|D|^{\frac{n-1}{n}}+C \varepsilon \log ^{2}(1+n) L_{D},
$$

where $C$ is an absolute constant.

Proof : By approximation ([S2, Th. 3.3.1] and [GZ, Section 5]), we can assume that $D$ has infinitely smooth support function and both $D$ and $K$ have infinitely smooth curvature functions. By (8), the condition (10) can be written as

$$
-\frac{1}{\pi}\left(f_{K} \cdot r^{-n-1}\right)^{\wedge}(\xi) \leq-\frac{1}{\pi}\left(f_{D} \cdot r^{-n-1}\right)^{\wedge}(\xi)+\varepsilon, \quad \forall \xi \in S^{n-1} .
$$

By $(7),\left(h_{D} \cdot r\right)^{\wedge} \leq 0$ on the sphere $S^{n-1}$. Therefore, integrating (11) with respect to a negative density, we get

$$
\begin{gathered}
\int_{S^{n-1}}\left(h_{D} \cdot r\right)^{\wedge}(\xi)\left(f_{D} \cdot r^{-n-1}\right)^{\wedge}(\xi) d \xi \\
\geq \int_{S^{n-1}}\left(h_{D} \cdot r\right)^{\wedge}(\xi)\left(f_{K} \cdot r^{-n-1}\right)^{\wedge}(\xi) d \xi+\pi \varepsilon \int_{S^{n-1}}\left(h_{D} \cdot r\right)^{\wedge}(\xi) d \xi .
\end{gathered}
$$

Using this, (2), (4), Parseval's formula (9) and the first Minkowski inequality (3),

$$
\begin{gathered}
(2 \pi)^{n} n|D|=(2 \pi)^{n} \int_{S^{n-1}} h_{D}(x) f_{D}(x) d x \\
\geq(2 \pi)^{n} \int_{S^{n-1}} h_{D}(x) f_{K}(x) d x+\pi \varepsilon \int_{S^{n-1}}\left(h_{D} \cdot r\right)^{\wedge}(\xi) d \xi . \\
\geq(2 \pi)^{n} n|D|^{\frac{1}{n}}|K|^{\frac{n-1}{n}}+\pi \varepsilon \int_{S^{n-1}}\left(h_{D} \cdot r\right)^{\wedge}(\xi) d \xi
\end{gathered}
$$

The result follows from Lemma 2 .

Now we show that if the projection body condition is dropped, the result may go in the opposite direction. 
Proposition 2. Suppose that $K$ is an origin-symmetric convex body in $\mathbb{R}^{n}$, which is not a projection body. Suppose also that $h_{K}, f_{K} \in$ $C^{\infty}\left(S^{n-1}\right)$, and $f_{K}$ is strictly positive on $S^{n-1}$. Then for small enough $\varepsilon>0$ there exists an origin-symmetric convex body $D$ in $\mathbb{R}^{n}$ so that

$$
P_{D}(\theta) \leq P_{K}(\theta) \leq P_{D}(\xi)+\varepsilon, \quad \forall \theta \in S^{n-1},
$$

but

$$
|K|^{\frac{n-1}{n}} \geq|D|^{\frac{n-1}{n}}+c_{n} \varepsilon
$$

Proof : By (7), since $K$ is not a projection body, there exists a symmetric open set $\Omega \subset S^{n-1}$, where $\left(h_{K} \cdot r\right)^{\wedge}>0$. Let $v$ be an even infinitely smooth non-negative function supported in $\Omega$. Extend $v$ to a homogeneous function $v \cdot r$ of degree 1 on $\mathbb{R}^{n}$. The Fourier transform of $v \cdot r$ is a homogeneous of degree $-n-1$ function $g \cdot r^{-n-1}$, where $g$ is an infinitely smooth function on the sphere; recall (6).

Define an even function $h$ on the sphere $S^{n-1}$ by

$$
f_{K}=h+\delta g+\frac{\varepsilon}{\left|B_{2}^{n-1}\right|} .
$$

Choose $\varepsilon, \delta>0$ small enough so that $h>0$ everywhere on $S^{n-1}$ (recall that $f_{K}>0$ ). By the Minkowski existence theorem (see, for example, [S2, Section 7.1]), there exists an origin-symmetric convex body $D$ in $\mathbb{R}^{n}$, whose curvature function $f_{D}=h$. Extend the functions in the definition of $h$ to even homogeneous of degree $-n-1$ functions on $\mathbb{R}^{n}$ :

$$
f_{K} \cdot r^{-n-1}=f_{D} \cdot r^{-n-1}+\delta g \cdot r^{-n-1}+\frac{\varepsilon}{\left|B_{2}^{n-1}\right|} \cdot r^{-n-1} .
$$

By $(8),\left(r^{-n-1}\right)^{\wedge}(\theta)=\left(f_{2} \cdot r^{-n-1}\right)^{\wedge}(\theta)=-\pi\left|B_{2}^{n-1}\right|$ for every $\theta \in S^{n-1}$ (here $f_{2} \equiv 1$ is the curvature function of the unit Euclidean ball).

Taking the Fourier transform of both sides of (12) and again using (8), we get

$$
\left(f_{K} \cdot r^{-n-1}\right)^{\wedge}(\theta)=\left(f_{D} \cdot r^{-n-1}\right)^{\wedge}(\theta)+(2 \pi)^{n} \delta v(\theta)-\pi \varepsilon, \quad \forall \theta \in S^{n-1},
$$

and

$$
-\pi P_{K}(\theta)=-\pi P_{D}(\theta)+(2 \pi)^{n} \delta v(\theta)-\pi \varepsilon, \quad \forall \theta \in S^{n-1} .
$$

Since $v \geq 0$, the latter implies

$$
P_{K}(\theta) \leq P_{D}(\theta)+\varepsilon, \quad \forall \theta \in S^{n-1} .
$$

Also, choosing $\delta$ small enough, we can assure that $P_{K}(\theta) \geq P_{D}(\theta)$ for every $\theta \in S^{n-1}$. 
On the other hand, multiplying (13) by $\left(h_{K} \cdot r\right)^{\wedge}(\theta)$, integrating over the sphere, and using the fact that $v \geq 0$ is supported in $\Omega$, where $\left(h_{K} \cdot r\right)^{\wedge}>0$, we get

$$
\begin{gathered}
\int_{S^{n-1}}\left(h_{K} \cdot r\right)^{\wedge}(\theta)\left(f_{K} \cdot r^{-n-1}\right)^{\wedge}(\theta) d \theta \\
=\int_{S^{n-1}}\left(h_{K} \cdot r\right)^{\wedge}(\theta)\left(f_{D} \cdot r^{-n-1}\right)^{\wedge}(\theta) d \theta \\
+(2 \pi)^{n} \delta \int_{S^{n-1}} v(\theta)\left(h_{K} \cdot r\right)^{\wedge}(\theta) d \theta-\pi \varepsilon \int_{S^{n-1}}\left(h_{K} \cdot r\right)^{\wedge}(\theta) d \theta \\
\geq \int_{S^{n-1}}\left(h_{K} \cdot r\right)^{\wedge}(\theta)\left(f_{D} \cdot r^{-n-1}\right)^{\wedge}(\theta) d \theta-\pi \varepsilon \int_{S^{n-1}} \widehat{h_{K}}(\theta) d \theta .
\end{gathered}
$$

Now, by Parseval's formula (9), (2), (4) and the first Minkowski inequality,

$$
\begin{gathered}
(2 \pi)^{n} n|K| \geq(2 \pi)^{n} \int_{S^{n-1}} h_{K}(\theta) f_{D}(\theta) d \theta-\pi \varepsilon \int_{S^{n-1}}\left(h_{K} \cdot r\right)^{\wedge}(\theta) d \theta \\
\geq(2 \pi)^{n} n|K|^{\frac{1}{n}}|D|^{\frac{n-1}{n}}-\pi \varepsilon \int_{S^{n-1}}\left(h_{K} \cdot r\right)^{\wedge}(\theta) d \theta .
\end{gathered}
$$

The result follows from Lemma 1.

The following separation result will be used to prove Theorem 4 .

Proposition 3. Suppose that $K$ is an origin-symmetric convex body in $\mathbb{R}^{n}$ with strictly positive curvature which is not a projection body and is a dilate of an isotropic body. Also suppose that the support and curvature functions of $K$ are infinitely smooth. Then for small enough $\varepsilon>0$ there exists an origin-symmetric convex body $D$ in $\mathbb{R}^{n}$ so that

$$
P_{K}(\theta) \leq P_{D}(\theta)-\varepsilon, \quad \forall \theta \in S^{n-1},
$$

but

$$
|D|^{\frac{n-1}{n}} \leq|K|^{\frac{n-1}{n}}+C \log ^{2}(1+n) L_{K} \varepsilon,
$$

where $C$ is an absolute constant.

Proof : The proof follows the steps of the proof of Theorem 2. Define the functions $v$ and $g$ in the same way. Then define a body $D$ by

$$
f_{D}=f_{K}-\delta g+\frac{\varepsilon}{\left|B_{2}^{n-1}\right|} .
$$

At the very end use Lemma 2 instead of Lemma 1. 


\section{VOLUME DIFFERENCE AND HYPERPLANE INEQUALITIES}

In this section we apply stability theorems to prove our main results. We start with the volume difference inequality of Theorem 1.

Theorem 1. Let $D$ be a projection body in $\mathbb{R}^{n}$ in isotropic position up to a dilation, and let $K$ be any origin-symmetric convex body in $\mathbb{R}^{n}$. Suppose that there exists $\xi \in S^{n-1}$ so that $P_{K}(\xi)>P_{D}(\xi)$. Then

$$
\max _{\xi \in S^{n-1}}\left(P_{K}(\xi)-P_{D}(\xi)\right) \geq \frac{c}{\log ^{2} n}\left(|K|^{\frac{n-1}{n}}-|D|^{\frac{n-1}{n}}\right),
$$

where $c$ is an absolute constant.

Proof : Let $\varepsilon=\max _{\xi \in S^{n-1}}\left(P_{K}(\xi)-P_{D}(\xi)\right)$. By the condition of Theorem $1, \varepsilon>0$. Now we can apply Proposition 1 to $K, D, \varepsilon$. We get

$$
|K|^{\frac{n-1}{n}} \leq|D|^{\frac{n-1}{n}}+C \log ^{2}(1+n) L_{D} \max _{\xi \in S^{n-1}}\left(P_{K}(\xi)-P_{D}(\xi)\right),
$$

where $C$ is an absolute constant. The result follows from the fact that isotropic constants of projection bodies (zonoids) are uniformly bounded by an absolute constant; see [MP, p.96].

"Differentiating" the inequality of Theorem 1, we prove a hyperplane inequality for the surface area of projection bodies.

Theorem 2. Suppose that $D$ is a projection body in $\mathbb{R}^{n}$ in isotropic position up to a dilation (see definition in Section 2). Then

$$
S(D) \leq C \log ^{2} n \max _{\xi \in S^{n-1}} S\left(D \mid \xi^{\perp}\right)|D|^{\frac{1}{n}}
$$

where $C$ is an absolute constant.

Proof : The surface area of $D$ can be computed as

$$
S(D)=\lim _{\varepsilon \rightarrow+0} \frac{\left|D+\varepsilon B_{2}^{n}\right|-|D|}{\varepsilon} .
$$

The inequality (14) with the bodies $K=D+\varepsilon B_{2}^{n}$ and $D$ implies

$$
\frac{\left|D+\varepsilon B_{2}^{n}\right|^{\frac{n-1}{n}}-|D|^{\frac{n-1}{n}}}{\varepsilon} \leq C \log ^{2} n \max _{\xi \in S^{n-1}} \frac{\left|\left(D \mid \xi^{\perp}\right)+\varepsilon B_{2}^{n-1}\right|-|D| \xi^{\perp} \mid}{\varepsilon}
$$

where $C$ is an absolute constant.

By the Minkowski theorem on mixed volumes ([S2, Theorem 5.1.6] or [G, Theorem A.3.1]),

$$
\frac{\left|\left(D \mid \xi^{\perp}\right)+\varepsilon B_{2}^{n-1}\right|-|D| \xi^{\perp} \mid}{\varepsilon}=\sum_{i=1}^{n-1}\left(\begin{array}{c}
n-1 \\
i
\end{array}\right) W_{i}\left(D \mid \xi^{\perp}\right) \varepsilon^{i-1},
$$


where $W_{i}$ are quermassintegrals. The function $\xi \mapsto D \mid \xi^{\perp}$ is continuous from $S^{n-1}$ to the class of origin-symmetric convex sets equiped with the Hausdorff metric, and $W_{i}$ 's are also continuous with respect to this metric (see [S2, p.275]), so the functions $\xi \mapsto W_{i}\left(D \mid \xi^{\perp}\right)$ are continuous and, hence, bounded on the sphere. This implies that the left-hand side of (17) converges to $S\left(D \mid \xi^{\perp}\right)$, as $\varepsilon \rightarrow 0$, uniformly with respect to $\xi$. The latter allows to switch the limit and maximum in the right-hand side of (16), as $\varepsilon \rightarrow 0$. Sending $\varepsilon$ to zero in (16), we get

$$
\frac{n-1}{n}|D|^{-1 / n} S(D) \leq C \log ^{2} n \max _{\xi \in S^{n-1}} S\left(D \mid \xi^{\perp}\right) .
$$

Theorems 3 and 4 follow from Propositions 2 and 3 by putting $\varepsilon=\max _{\xi \in S^{n-1}}\left(P_{K}(\xi)-P_{D}(\xi)\right)$ and $\varepsilon=\min _{\xi \in S^{n-1}}\left(P_{D}(\xi)-P_{K}(\xi)\right)$, correspondingly.

Theorem 3. Suppose that $K$ is an origin-symmetric convex body in $\mathbb{R}^{n}$ with strictly positive curvature that is not a projection body. Then there exists an origin-symmetric convex body $D$ in $\mathbb{R}^{n}$ so that $P_{K}(\xi) \geq P_{D}(\xi)$ for all $\xi \in S^{n-1}$ and

$$
\max _{\xi \in S^{n-1}}\left(P_{K}(\xi)-P_{D}(\xi)\right) \leq \frac{1}{c_{n}}\left(|K|^{\frac{n-1}{n}}-|D|^{\frac{n-1}{n}}\right) .
$$

Theorem 4. Suppose that $K$ is an origin-symmetric convex body in $\mathbb{R}^{n}$ that is not a projection body and is in isotropic position up to a dilation, with isotropic constant $L_{K}$. Then there exists an origin-symmetric convex body $D$ in $\mathbb{R}^{n}$ so that $P_{D}(\xi) \geq P_{K}(\xi)$ for all $\xi \in S^{n-1}$ and

$$
\min _{\xi \in S^{n-1}}\left(P_{D}(\xi)-P_{K}(\xi)\right) \geq \frac{c}{L_{K} \log ^{2} n}\left(|D|^{\frac{n-1}{n}}-|K|^{\frac{n-1}{n}}\right),
$$

where $c$ is an absolute constant.

Remark. Putting $D=\beta B_{2}^{n}$ in (14) and sending $\beta \rightarrow 0$, we get a hyperplane inequality for volume

$$
\max _{\xi \in S^{n-1}} P_{K}(\xi) \geq \frac{c}{\log ^{2} n}|K|^{\frac{n-1}{n}} .
$$

However, a stronger inequality

$$
|L|^{\frac{n-1}{n}} \leq c_{n} \max _{\xi \in S^{n-1}}|L| \xi^{\perp} \mid
$$

holds for all origin-symmetric convex bodies and follows from the Cauchy projection formula for the surface area and the classical isoperimetric inequality; see [G, p. 363]. 
It can be deduced directly from the solution to Shephard's problem (see $\left[G\right.$, Corollary 9.3.4]) that, if $L$ is a projection body in $\mathbb{R}^{n}$, then

$$
|L|^{\frac{n-1}{n}} \geq c_{n} \min _{\xi \in S^{n-1}}|L| \xi^{\perp} \mid .
$$

Recall that $c_{n}>1 / \sqrt{e}$. For general symmetric convex bodies, Ball [Ba] proved that $c_{n}$ may and has to be replaced in (18) by $c / \sqrt{n}$, where $c$ is an absolute constant.

Acknowledgement. I wish to thank the US National Science Foundation for support through grant DMS-1265155. Part of the work was done during my stay at the Max Planck Institute for Mathematics in Bonn in Spring 2015.

\section{REFERENCES}

[A] A. D. Aleksandrov, On the surface area function of a convex body, Mat. Sb. 6 (1939), 167-174.

[Ba] K. Ball, Shadows of convex bodies, Trans. Amer. Math. Soc. 327 (1991), 891901.

[Bl] E. D. Bolker, A class of convex bodies, Trans. Amer. Math. Soc. 145 (1969), 323-345.

[Bo] J. Bourgain, On the distribution of polynomials on high-dimensional convex sets, Geometric aspects of functional analysis, Israel seminar (1989-90), Lecture Notes in Math. 1469 Springer, Berlin, 1991, 127-137.

[BGVV] S. Brazitikos, A. Giannopoulos, P. Valettas and B. Vritsiou, Geometry of isotropic log-concave measures, Amer. Math. Soc., Providence, RI, 2014.

[G] R. J. Gardner, Geometric tomography, Second edition, Cambridge University Press, Cambridge, 2006.

[GZ] E. Grinberg and Gaoyong Zhang, Convolutions, transforms, and convex bodies, Proc. London. Math. Soc. (3) 78 (1999), 77-115.

[Kl] B. Klartag, On convex perturbations with a bounded isotropic constant, Geom. Funct. Anal. 16 (2006), 1274-1290.

[K1] A. Koldobsky, Fourier analysis in convex geometry, Amer. Math. Soc., Providence RI, 2005.

[K2] A. Koldobsky, Stability in the Busemann-Petty and Shephard problems, Adv. Math. 228 (2011), 2145-2161.

[K3] A. Koldobsky, Stability and separation in volume comparison problems, Math. Model. Nat. Phenom. 8 (2013), 156-169.

[KRZ] A. Koldobsky, D. Ryabogin and A. Zvavitch, Projections of convex bodies and the Fourier transform, Israel J. Math. 139 (2004), 361-380.

[M] E. Milman, On the mean-width of isotropic convex bodies and their associated $L_{p}$-centroid bodies, arxiv:1402.0209v1.

[MP] V. Milman and A. Pajor, Isotropic position and inertia ellipsoids and zonoids of the unit ball of a normed n-dimensional space, in: Geometric Aspects of Functional Analysis, ed. by J. Lindenstrauss and V. Milman, Lecture Notes in Mathematics 1376, Springer, Heidelberg, 1989, pp. 64-104. 
[Pe] C. M. Petty, Projection bodies, Proc. Coll. Convexity (Copenhagen 1965), Kobenhavns Univ. Mat. Inst., 234-241.

[S1] R. Schneider, Zu einem problem von Shephard über die projektionen konvexer körper, Math. Z. 101 (1967), 71-82.

[S2] R. Schneider, Convex bodies: The Brunn-Minkowski theory, Cambridge Univ. Press, Cambridge, 1993.

[Sh] G. C. Shephard, Shadow sysytems of convex bodies, Israel J. Math. 2 (1964)' 229-306.

Department of Mathematics, University of Missouri, Columbia, MO 65211

E-mail address: koldobskiya@missouri.edu 